\title{
Prevalence of Sjögren's syndrome in a closed rural community
}

\author{
Urania G Dafni, Athanasios G Tzioufas, Panayiotis Staikos, Fotini N Skopouli, \\ Haralampos M Moutsopoulos
}

\begin{abstract}
Objective-To define the prevalence of Sjögren's syndrome (SS) through an epidemiological survey in a closed rural community. The classification of SS is based on the validated criteria reported by a multicentre study performed in Europe and supported by the Epidemiology Committee of the European Community (EEC-COMAC Epidemiology).

Methods-The population under study consisted of 837 women aged 18 years or older, residing in the Astakos community of Aitoloakarnania, Greece. The study protocol was subdivided in two parts. In part I, an exhaustive epidemiological survey of these women was conducted in July and August of 1992. The validated questionnaire used in the survey assesses both ocular and oral involvement. In part II, 45 of the women reporting symptoms of both dry eye and dry mouth were approached for a full examination based on the validated set of classification criteria of SS. The full complement of the diagnostic tests was performed on 35 of these women. A subject is classified as a definite primary SS case if at least four of six items of the subject's test items are positive. If three of six items are positive the subject is classified as a probable primary SS case.
\end{abstract}

Results-The classification criteria for definite primary SS were satisfied by five women. This number corresponds to an estimated prevalence of $0.60 \%$ (exact $95 \%$ CI $0.19 \%, 1.39 \%$ ). Probable primary SS was diagnosed for 25 women (prevalence $=2.99 \%$ ).

Conclusion-Because of the loss of follow up (10 of 45) and the use of slightly stricter criteria for inclusion of possible SS cases in part II of the study, we consider our estimate of the prevalence of SS to be conservative. This study concurring with other recent reports, suggests that SS is more prevalent than previously thought.

(Ann Rheum Dis 1997;56:521-525)

Sjögren's syndrome (SS) is a chronic autoimmune disease affecting primarily the exocrine glands of women and presents with xerostomia, keratoconjunctivitis sicca, and parotid gland enlargement. It can occur alone (primary SS) or in association with almost all of the systemic autoimmune rheumatic diseases (secondary SS). ${ }^{1}$ In rheumatology clinics, primary SS may appear as commonly as systemic lupus erythematosus. Furthermore, approximately $25 \%$ of rheumatoid arthritis and systemic lupus erythematosus patients present at least histological evidence of SS. ${ }^{1}$ Given that rheumatoid arthritis affects $0.2-5.3 \%$ of the general population, ${ }^{2} \mathrm{SS}$ can be considered a frequent medical problem. Among people without autoimmune connective tissue disease, necropsy studies have shown approximately $2-3 \%$ of people with focal infiltrates of the labial minor salivary gland compatible with SS. ${ }^{3}$

Studies on the prevalence of primary SS in the general population are limited mainly because of the lack of uniform classification criteria for the disease, differences in diagnostic techniques, as well as differences in the reference values of the tests used for the evaluation of KCS and salivary gland involvement. $^{4}$

The aim of this study was to estimate the prevalence of primary SS using (1) a well defined set of classification criteria for primary SS and (2) an accessible closed population. The criteria selected for the study are the recently reported classification criteria developed and validated with the support of the Epidemiology Committee of the European Community. ${ }^{5-7}$

\section{Methods}

STUDY POPULATION

As SS is a disease that mainly affects women, the population under study consisted of women at least 18 years of age residing in the Astakos community of Aitoloakarnania, Greece in June 1992. The 1971 national population survey reports 902 women of the relevant age group residing in Astakos while 837 resided there in 1992 at the time of our epidemiological survey. This is a closed community with minimal migration during the past 30 years. It is a typical Greek rural community with a total population of 2500 . The town hall records were the source of the addresses and birthdates for the subjects. The local general practitioner (PS) helped coordinate the administration of the questionnaires. Women with rheumatoid arthritis, systemic lupus erythematosus, scleroderma or other autoimmune rheumatic disorders were excluded from the study based on the American Rheumatism Association criteria. ${ }^{89}$ The level of collaboration by the targeted population of local women was exceptional.

The age distribution of the eligible women of Astakos was compared with that of the region 
of Aitoloakarnania excluding Astakos (table 1). The null hypothesis of identical age distribution in the two areas is not rejected ( $p$ value $=0.24)$. However, in the region of Aitoloakarnania excluding Astakos, a higher percentage of women in their forties and fifties $(29.3 \% v 37.7 \%)$ is observed. This is the peak incidence age for $\mathrm{SS}^{10}$ and it is underrepresented in the community of Astakos ( $\mathrm{p}$ value < $0.001)$.

\section{STUDY PROTOCOL}

The study protocol was designed based on the recently reported 'preliminary classification criteria' for SS. ${ }^{5}$ These criteria were developed with the support of the Epidemiology Committee of the European Community (EEC-COMAC Epidemiology) and were validated in a multicentre study conducted in 12 European countries. ${ }^{67}$ This study's protocol was subdivided in two parts. In part I, an exhaustive epidemiological survey of the targeted population was conducted during July and August of 1992. A simple six item questionnaire was given to the eligible women for assessing both ocular and oral involvement. ${ }^{5}$ The first three questions relate to dryness of the eye with the remaining three to dryness of the mouth symptoms. A member of the investigative team (PS) visited the women at their home, more than once if necessary. Information on comorbidities and related treatment was collected on all women who answered positively to at least one question either of dryness in the mouth or of dryness in the eye. Twenty eight women were either taking anti-hypertensive medication, or taking anti-depressants or medication for diabetes. One was receiving treatment for sarcoidosis.

In part II, the women reporting symptoms of dryness on both eye and mouth were asked to visit within a month, the Health Centre of Astakos for a more detailed evaluation. During these visits, a full clinical examination, tests providing objective evidence of ocular involvement and of salivary gland involvement as well as serum tests for the presence of autoantibodies were performed to rule out other causes of xerostomia. The tests were based on the validated set of classification criteria of SS. ${ }^{5} \mathrm{~A}$ subject is classified as a definite primary SS case if at least four of the six items of the subject's tests are positive. If three of the six items are positive the subject is classified as a probable primary SS case.

Table 1 Age distibution in the community of Astakos and the remainder of the region of Aitoloakarnania

\begin{tabular}{llllll}
\hline & \multicolumn{2}{c}{ Astakos } & & \multicolumn{2}{c}{ Region of Aitoloakarnania } \\
\cline { 2 - 3 } \cline { 5 - 6 } Age category & Number & $\%$ & & Number & $\%$ \\
\hline $18-30$ & 203 & 24.3 & & 15073 & 20.3 \\
$31-40$ & 144 & 17.2 & & 11371 & 15.3 \\
$41-50$ & 105 & 12.5 & & 14979 & 20.1 \\
$51-60$ & 140 & 16.7 & & 13085 & 17.6 \\
$61-70$ & 127 & 15.2 & & 9281 & 12.5 \\
$71-80$ & 91 & 10.9 & & 7187 & 9.7 \\
$81-90$ & 27 & 3.2 & & 3417 & 4.6 \\
Total & 837 & & & 74393 & \\
\cline { 5 - 6 } & & & &
\end{tabular}

Kruskal-Wallis $\mathrm{p}$ value for testing identity of age distribution $=0.24$
According to the 'preliminary classification criteria' women with either ocular alone symptoms or oral alone symptoms are still candidates for SS classification. ${ }^{5}$ The following more strict criterion was used in the study protocol: women who reported only symptoms of dryness of the eye or of the mouth but not of both were classified as non-SS. These women did not advance to the second part of the study and were not subjected to all the examinations. It is evident, that the exclusion criterion applied in this study results in more women being classified as non-SS. In addition, only one objective test, the unstimulated salivary flow test, was used for the objective determination of salivary gland involvement. The test was considered positive if the flow was less than or equal to $1.5 \mathrm{ml}$ in 15 minutes. The salivary scintigraphy and the parotid sialography tests suggested by Vitali et $a l^{5}$ were not performed in this study.

Objective evidence of ocular involvement was determined on the basis of a positive result for at least one of the following two tests: (a) Schirmer-I test ( $\leqslant=5 \mathrm{~mm}$ in 5 minutes) (b) rose bengal score ( $\geqslant=4$, according to the Van Bijsterveld scoring system). ${ }^{5}$ The histopathological features were defined based on a focus score $\geqslant=1$ on minor salivary gland biopsy (focus defined as an agglomeration of at least 50 mononuclear cells; focus score defined as the number of foci per $4 \mathrm{~mm}^{2}$ of glandular tissue). ${ }^{5}$ The serum antibodies to Ro/SS-A and $\mathrm{La} / \mathrm{SS}-\mathrm{B}$ were tested by counterimmunoelectrophoresis, ${ }^{11}$ antinuclear antibodies by indirect immunofluorescence using as substrate Hep-2 cells (Kallestad-USA), and the rheumatoid factor by Latex fixation (BoehringGermany). The cut off point for these two assays was 1:80 and $40 \mathrm{IU} / \mathrm{ml}$, respectively.

STATISTICAL ANALYSIS

The Kruskal-Wallis test was used to test for the hypothesis of identical distributions. Fisher's exact test was used to test for the difference in the percentage of women in the peak incidence age between Astakos and the remainder of Aitoloakarnania. A two sided exact confidence interval was constructed for the prevalence estimate. The statistical procedures were performed using the SAS and StatXact statistical packages. ${ }^{1213}$

\section{Results}

Part I: One hundred per cent of the targeted population completed the first part of the study. The six item questionnaire for determination of dry eyes and dry mouth symptoms was completed by all 837 eligible women. This was achieved through several home visits by the local medical practitioner (PS). Figure 1 is a histogram of the results by age category. Fourteen per cent (116 women) answered positively to at least one question either of dryness in the mouth or of dryness in the eye. This proportion is low compared with the results of other studies. ${ }^{14}$ It increases with age as depicted in figure 1. Eighty per cent of the positive answers related to only three of the questions; most common $(35.7 \%$ of the 


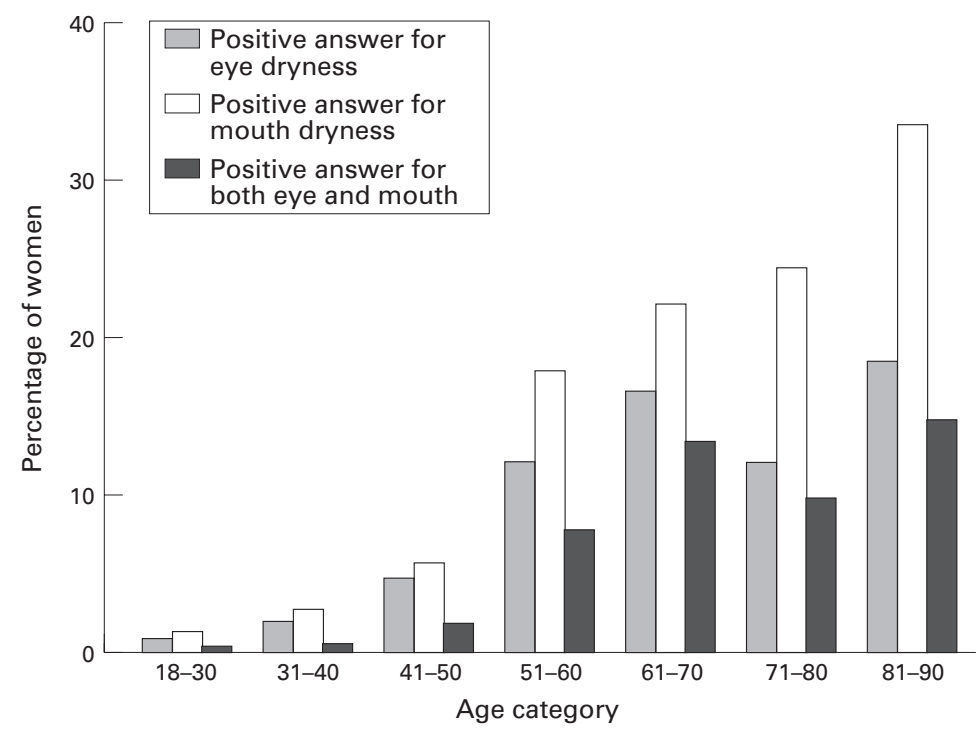

Figure 1 Percentage of the 837 women answering positively to at least one question for either eye or mouth dryness, by age category.

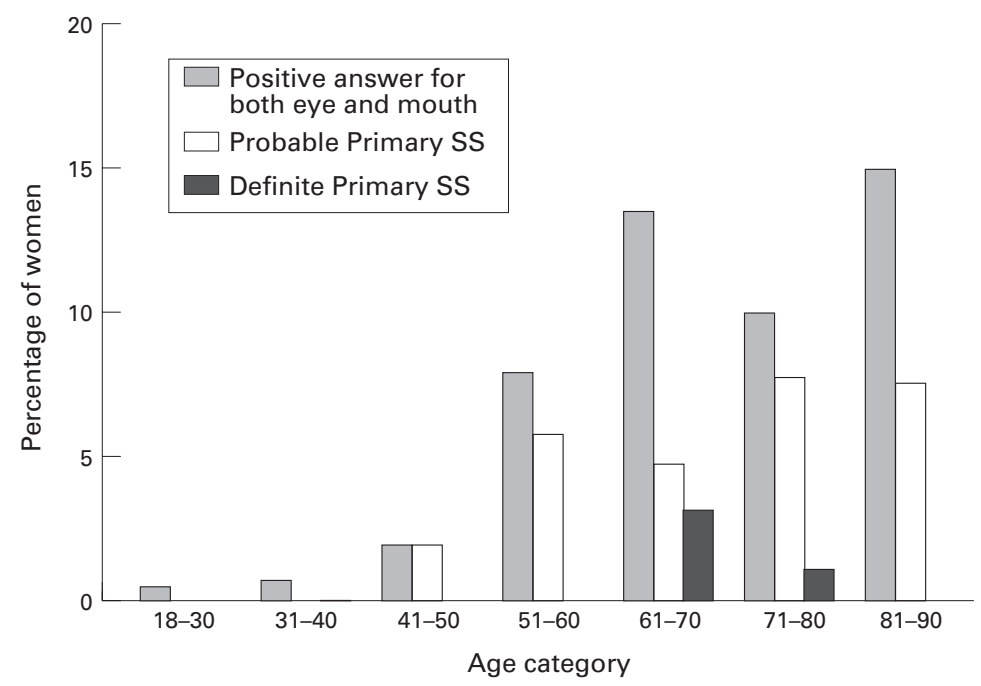

Figure 2 Percentage of women eligible for Part II and diagnosed with definite or probable primary SS, by age category.

positive answers) was the symptom of a daily feeling of dry mouth for more than three months, which was reported by $9.6 \%$ of the total population of 837 women; the second most common positive answer, reported by $6.9 \%$ of the women, was of a recurrent sensation of sand or gravel in the eyes $(25.9 \%$ of the positive answers); the third $(19.2 \%$ of the positive answers) was the frequent drinking of liquids to aid in swallowing dry foods, reported by $5.1 \%$ of the total population.

Fifty two women $(44.8 \%$ of the 116) answered positively only to questions relating to dryness of the mouth while 19 (16.4\%) reported exclusively dryness of the eye. Forty five women $(38.8 \%)$ answered positively to at least one question for the dryness of the eye and one for the dryness of the mouth and were asked to participate in Part II of the study. From these, two answered positively to all six questions, with a total of 16 women answering positively to at least four of the six questions. Ten of 45 women refused to sign the informed consent and participate in further examination.

Part II: The full complement of the diagnostic tests was performed on 35 of 45 eligible women. The Schirmer-I test was negative for all subjects. The rose bengal score and the minor salivary gland biopsy were positive for three and four subjects respectively. These were among the women classified as definite primary SS cases. The unstimulated salivary flow test was positive for six women, three of whom were classified as definite SS. Antinuclear antibodies were present at the serum of 26 women $(74.3 \%$ of the 35$)$. Rheumatoid factor and anti-Ro/SS-A antibodies were present in only four and one respectively, all of which were definite primary SS cases. No anti-La/SS-B antibody was detected.

Five women, four aged in their sixties and one 75 years old, satisfied four of the six criteria for the classification of definite primary SS. One of the five definite SS cases had a sister diagnosed with rheumatoid arthritis. No other definite SS case had any family history of autoimmune disease. Table 2 presents the results of the clinical and laboratory tests for the subjects classified as definite SS cases. An additional 25 women satisfied three of the six criteria and are classified as probable primary SS. Their age distribution is as follows; two in their forties, eight in their fifties, six in their sixties, seven in their seventies, and two over 80 . Histograms by age category, comparing the per cent diagnosed with definite and probable primary SS with the per cent answering positively to questions on both eye and mouth dryness, are presented in figure 2 .

The estimated prevalence of definite primary SS is $0.60 \%$ (exact $95 \%$ CI $0.19 \%$, $1.39 \%)$. Probable primary SS prevalence is

Table 2 Clinical and laboratory characteristics for definite primary SS cases

\begin{tabular}{|c|c|c|c|c|c|c|c|c|c|c|}
\hline Patient & Age & $\begin{array}{l}\text { Duration of } \\
\text { Sicca } \\
\text { symptoms (y) }\end{array}$ & $\begin{array}{l}\text { Tear or saliva } \\
\text { substitutes }(y)\end{array}$ & $\begin{array}{l}\text { Rose bengal score } \\
\text { (Van Bisterveld's } \\
\text { scoring system) }\end{array}$ & $\begin{array}{l}\text { Minor salivary } \\
\text { gland biopsy } \\
\text { (Tarpley's } \\
\text { classification) }\end{array}$ & $\begin{array}{l}\text { Unstimulated } \\
\text { salivary flow } \\
\text { test (ml) }\end{array}$ & ANA (titre/type) & $\begin{array}{l}R F \\
(I U / m l)\end{array}$ & $\begin{array}{l}\text { Anti-Rol } \\
\text { SSA }\end{array}$ & $\begin{array}{l}\text { Anti-La } \\
S S B\end{array}$ \\
\hline 1 & 61 & 5 & 1 & $\begin{array}{l}\text { LE } 3 / 9 \\
\text { RE } 4 / 9\end{array}$ & +3 & 2.2 & 1:640/fine spec & 160 & - & - \\
\hline 2 & 63 & 3 & - & $\begin{array}{c}\text { LE } 4 / 9 \\
\text { RE } 5 / 9\end{array}$ & +3 & 2.0 & $1: 1280 /$ fine spec & 640 & + & - \\
\hline 3 & 65 & 5 & - & $\begin{array}{l}\text { LE } 1 / 9 \\
\text { RE } 1 / 9\end{array}$ & +1 & 1.21 & - & - & - & - \\
\hline 4 & 66 & 18 & - & $\begin{array}{l}\mathrm{LE} 2 / 9 \\
\mathrm{RE} 2 / 9\end{array}$ & +3 & 0.7 & $1: 320 /$ speckled & 80 & - & - \\
\hline $5^{\star}$ & 75 & 23 & - & $\begin{array}{l}\mathrm{LE} 3 / 9 \\
\operatorname{RE} 4 / 9\end{array}$ & +3 & 1.3 & $1: 160 /$ speckled & 80 & - & - \\
\hline
\end{tabular}


estimated at $2.99 \%$ (exact $95 \%$ CI $1.94 \%$, $4.38 \%)$. Thus, an estimated prevalence of $3.59 \%$ (exact $95 \%$ CI $2.43 \%, 5.08 \%$ ) corresponds to the combined definite/probable primary SS.

\section{Discussion}

This study presents estimates of the prevalence of definite and probable SS. It is the first report in a closed rural community, capturing information on SS symptoms from the full population of 837 women over 18 years old. The tools used for the diagnosis of SS are based on the criteria suggested by the European concerted action. ${ }^{5}$ These criteria are well defined, and have been validated in a multicentre European study. ${ }^{67}$ The presence of four of six items of the criteria set, classifies subjects as definite SS. This rule, which was also used in our study, was shown to have good sensitivity $(93.5 \%)$ and specificity $(94.0 \%)$. The sample of Astakos community could be regarded as representative of the general population of Greece regarding disease prevalence (Government morbidity and mortality reports similar to the rest of Greece). However, the percentage of women in their forties and fifties is smaller in Astakos than in the region of Aitoloakarnania.

We consider our estimates of the prevalence of definite and probable primary SS as conservative for the following three reasons that affect them downwards: (1) we used slightly stricter criteria for inclusion of possible SS cases in Part II of the study, (2) the percentage of the women of Astakos in the peak incidence age was smaller than that of the surrounding region of Aitoloakarnania, and (3) 10 of 45 women eligible for full evaluation for SS refused to participate.

According to our results the disease is seen most often in the sixth decade of life, as four of five definite SS cases fell into that age category with the remaining one being 75 years old. Our results are in agreement with studies from Great Britain, Greece, and Sweden ${ }^{15} 161819$ suggesting that $2-5 \%$ of elderly patients have features of SS. In two of the studies ${ }^{1516}$ the affected subjects had positive objective tests for exocrine gland involvement but no antibodies to Ro/SS-A or La/SS-B. None of the SS cases in our study had either swollen salivary glands, or signs and symptoms of extraglandular manifestations. These women had mild complaints of dry eyes and mouth for several years but were not aware of the disease. The criteria used so far for the diagnosis of primary SS, presented high specificity but remarkably low sensitivity. Thus, patients diagnosed according to this set of criteria had expressed the full blown clinical picture of the disease.

In an elderly population, the reasons for dryness of the eye and mouth are several. The main ones are diabetes, use of certain drugs, as well as conjunctival or corneal pterygium, or both. In 10 of 35 women that were tested for ocular involvement, corneal pterygium was diagnosed. The high prevalence of pterygium can be explained by the proximity of Astakos to the sea and its high levels of sun exposure.
The most common symptom reported in Part I of the study was the daily feeling of dry mouth for more than three months (9.6\%), followed by the sensation of sand or gravel in the eyes $(6.9 \%)$. The most rare symptom was the use of tear substitutes more than three times a day $(0.6 \%)$, followed by the symptom of recurrent or persistently swollen salivary glands $(1.4 \%)$. These two less common symptoms were reported by only two of five definite SS cases. This suggests that they are not sensitive for primary SS. It is noteworthy that the other four symptoms were reported by all five SS patients.

In our study, the Schirmer-I test was not positive for any subject while the rose bengal score was positive in three of five SS cases $(60.0 \%)$. These findings do not agree with the report by Vitali et ap according to which, the Schirmer-I test is very sensitive and the rose bengal test more specific. It should be noted that a milder form of SS was present in the patients in our study, such that none of them had asked for medical help for their condition.

Our findings regarding the minor salivary gland biopsy, the unstimulated salivary flow test, ${ }^{17}$ and the presence of autoantibodies, is in agreement to Vitali et al. ${ }^{5}$ The antinuclear antibodies were present at the serum of 26 women ( $74.3 \%$ of the 35 examined), four of whom were definite SS cases. These results show that this serum antibody test is very sensitive but not highly specific. In our study the diagnosis of definite SS was based on the presence of autoantibodies (anti-Ro/SS-A) only in one of the five patients.

SS has been traditionally considered a rare disease. A recent epidemiological study ${ }^{18}$ estimates the prevalence of primary SS for a population with age ranging from 52 to 72 in the order of $2.7 \%$. In contrast, in our study, the total female adult population was examined and the prevalence of SS was estimated at $0.60 \%$. When considering the more restricted age range 51 to 70 , our estimate is lower than the one reported by Jacobsson et al, ${ }^{18}$ - that is, $1.5 \%$ (four definite SS cases among 267 in that age group). We view the estimated prevalence of this study, $0.60 \%$, as a representative estimate of the minimum prevalence of primary SS in the general female population.

Based on our study and the Swedish epidemiological study, ${ }^{18}$ each conducted in different areas of Europe, it seems that SS is a rather common condition, affecting at least $0.19 \%-1.39 \%$ (and possibly $2.43 \%-5.08 \%$ ) of the general population extending clinically from an overt disease (definite SS) to a subclinical state (probable SS).

1 Talal N, Moutsopoulos HM, Kassan SS. Sjogren's syndrome, clinical and immunological aspects. Berlin: Springer-Verlag, 1987.

2 Silman AJ. Epidemiology of the rheumatic diseases. In: Maddison PJ, Isenberg DA, Woo P, Glass DN, eds. Oxford textbook of rheumatology. Oxford: Oxford University Press, 1993: 499-513.

3 Scott J. Qualitative and quantitative observations on the histology of human labial salivary glands obtained postmortem. Journal of Biologic Buccale 1980;8:187-200.

mortem. Journal of Biologic Buccale 1980;8:187-200.
4 Vitali C, Bombardieri S. Diagnostic criteria for Sjogren's syndrome: the state of the art. Clin Exp Reumatol 1990;8 (suppl 5):13-6. 
5 Vitali C, Bombardieri S, Moutsopoulos HM, Balestrieri G, Bencivelli W, Bernstein R, et al. Preliminary criteria for the classification of Sjogren's syndrome. Results of an EEC prospective concerted action. Arthritis Rheum 1993 36:340-7

6 Vitali C, Moutsopoulos HM, Bombardieri S, and the European Community Study Group on Diagnostic Criteria for Siogren's syndrome. The European Community Study Group on Diagnostic Criteria for Sjogren's syndrome Sensitivity and specificity of tests for ocular and oral involvement in Sjogren's syndrome. Ann Rheum Dis 1994;53:637-47

7 Vitali C, Bombardieri S, Moutsopoulos HM, Coll J, Gerli R, Hatron $\mathrm{P}$ Y, et al. Validation of the preliminary classification criteria for Sjogren's syndrome. Results of prospective multicentre European study. Ann Rheum Dis 1996;55:116-21.

8 Tan EM, Cohen AS, Fries JF, Masi AT, McShane DJ, Rothfield NF, et al. The 1982 revised criteria for the classification of systemic lupus erythematosus. Arthritis Rheum 1982;25:1271-7.

9 Arnett FC, Edworthy SM, Bloch DA, McShane DJ, Fries JF, Cooper NS, et al. The American Rheumatism AssociaJF, Cooper NS, et al. The American Rheumatism Association 1987 revised criteria for the classification of
toid arthritis. Arthritis Rheum 1988;31:315-24.

10 Pavlidis NA, Karsh J, Moutsopoulos HM. The clinical picture of primary Sjogren's syndrome: A retrospective study. J Reumatol 1982;9:685-90.

11 Schur P, DeAngelis D, Jackson J. Immunological detection of nucleic acids and nuclear antigens by counterimmunoelectrophoresis. Clin Exp Immunol 1974;17:209-18.
12 SAS Institute Inc. SAS Procedures Guide. Release 6.03 edition. Cary NC: SAS Institute Inc, 1988.

13 StatXact. Cambridge, Massachusetts: CYTEL Software Corporation,, 1991.

14 Jacobsson LTH, Hansen BU, Manthorpe R, Hardgrave K, Neas B, Harley JB. Association of dry eyes and dry mouth with anti-Ro/SS-A and anti-La/SS-B autoantibodies in with anti-Ro/SS-A and anti-La/SS-B autoantibodies

15 Strickland RW, Tesar J, Berne B, Hobbs B, Lewis D, Welton R. The frequency of sicca syndrome in an elderly female population. J Rheumatol 1985;14:766-71.

16 Drosos AA, Andonopoulos AP, Costopoulos JS, Papadimitriou CS, Moutsopoulos HM. Prevalence of primary Sjogren's syndrome in an elderly population. $\mathrm{Br} \mathrm{J}$ Rheumatol 1988;27:123-7.

17 Skopouli FN, Siouna-Fatourou HI, Ziciadis C, Moutsopoulos HM. Evaluation of unstimulated whole saliva flow rate and stimulated parotid flow as confirmatory tests for xerostomia. Clin Exp Rheumatol 1989;7:127-9.

18 Jacobsson LTH, Axell TE, Hansen BU, Henriesoy VJ, Larsen A, Lieberkind K, et al. Dry eyes and mouth: an epidemiological study in Swedish adults, with special reference to primary Sjogren's syndrome. Jutoimmun 1989;2:521-7.

19 Whaley K, Williamson J, Wilson T, McGavin DDM, Hughes GRV, Hughes $\mathrm{H}$, et al. Sjogren's syndrome and autoimmunity in a geriatric population. Age Ageing 1972; $1: 197-206$

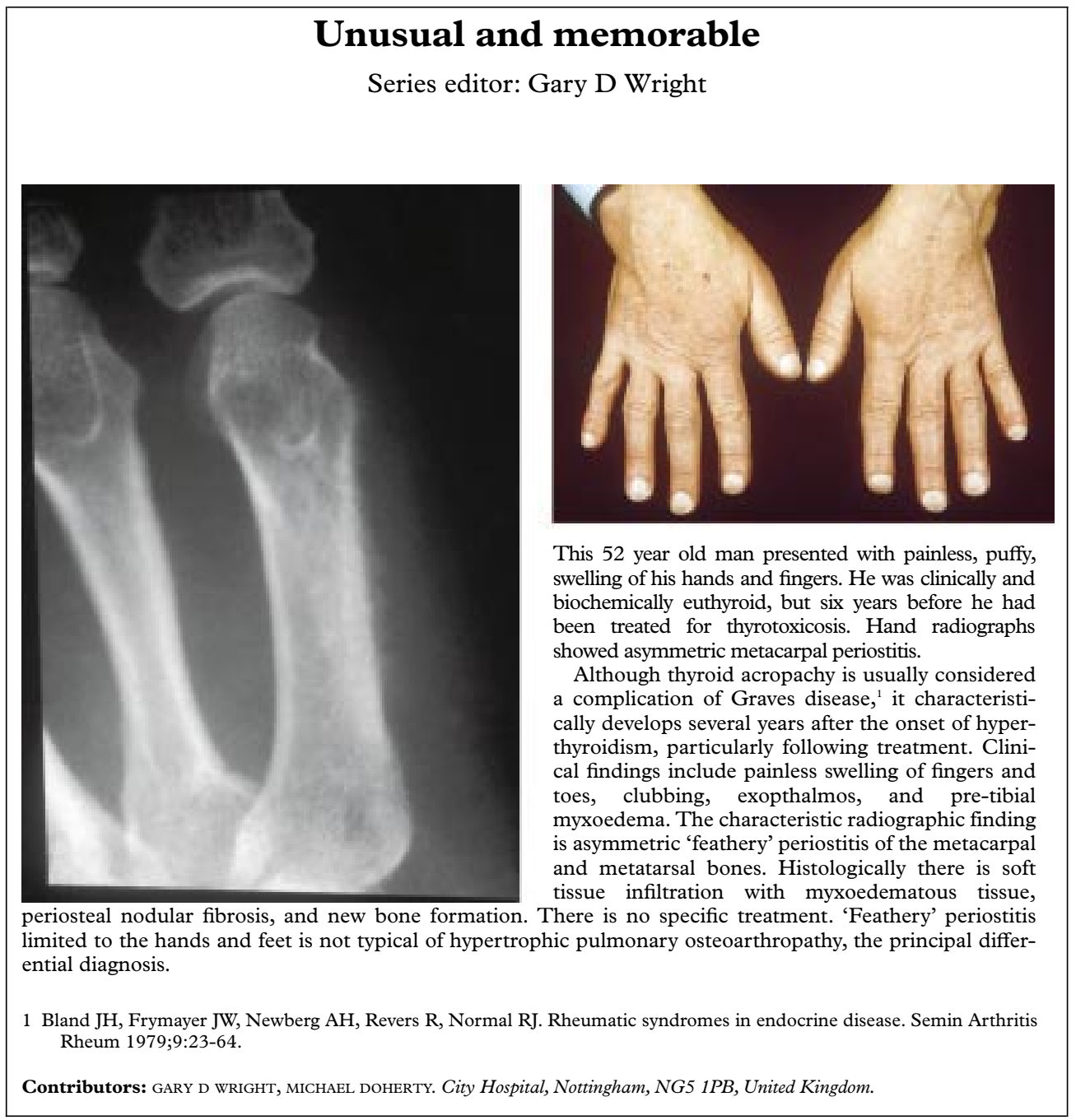

